Artículo

\title{
Racionalidad individual, legitimidad política, y factores socioeconómicos en Guatemala
}

\section{Individual rationality, political legitimacy, and socioeconomic factors in Guatemala}

\author{
José Ernesto Javier Duarte Madrid \\ Universidad de San Carlos de Guatemala \\ jed623@hotmail.com \\ https://orcid.org/0000-0003-1894-8820
}

\section{Referencia del artículo}

Duarte Madrid, J. E. J. (2021)._Racionalidad individual, legitimidad política, y factores socioeconómicos en Guatemala. Revista Guatemalteca de Cultura. 1(1), 17-26.

DOI: 10.46954/revistaguatecultura.v1i1.7

Recibido 15/09/ 2020

Aceptado 25/01/2021

\section{Resumen}

El presente estudio se hizo con base en la investigación cuantitativa, las teorías de la elección pública y racionalidad individual, además del estudio de cultura política de los guatemaltecos realizado por El Proyecto de Opinión Pública de América Latina (LAPOP), consistió en una muestra aleatoria estratificada de 1,596 encuestas, hechas a lo largo del país. Se exploró la legitimidad del sistema político guatemalteco y su relación con aspectos socioeconómicos. La pregunta general fue, ¿cómo se explica desde la racionalidad individual la correlación entre la variable factores 
socioeconómicos y legitimidad política en Guatemala, período 2019?, la investigación arrojó que los individuos de menor edad, menos años de escolaridad, con un fuerte arraigó religioso, así como los de menor ingreso apoyan el sistema político en su conjunto, no obstante, rechazan el accionar particular de las instituciones políticas, esa contradicción llevó a plantear la siguiente interrogante ¿por qué los ciudadanos se aferran a sistemas políticos ineficientes?, así, la respuesta se encontró en cuatro paradojas, de la información y la irracionalidad racional, paradoja fatalista, paradoja benevolente, y; paradoja de la intolerancia, bajo estos elementos se dedujo que los sistemas políticos democráticos tienen una imposibilidad parcial para satisfacer las preferencias de todos los individuos en una sola función de utilidad, generándose así un cíclico descontento ciudadano.

$\triangle$ Palabras clave: legitimidad política, racionalidad, individualismo metodológico, sistema político, política

\section{Abstract}

The present study was carried out using quantitative research and theories of public choice and individual rationality, it was based on the study of political culture of Guatemalans carried out by the Latin American Public Opinion Project (LAPOP), it consisted of a random sample stratified of 1,596 surveys, made throughout the country. Thus, the legitimacy of the Guatemalan political system and its relationship with socioeconomic aspects were explored. The general question was, how is the correlation between the variable socioeconomic factors and political legitimacy explained from individual rationality in Guatemala, period 2019? The research showed that younger individuals, fewer years of schooling, with a strong religious roots In the same way that those with lower income support the political system as a whole, however, they reject the particular actions of political institutions, this contradiction led to the following question: why do citizens cling to inefficient political systems? was found in four paradoxes, of information and rational irrationality, fatalistic paradox, benevolent paradox, and; Paradox of intolerance, under these premises it was deduced that democratic political systems have a partial impossibility to satisfy the preferences of all individuals in a single utility function, thus generating a cyclical discontent in citizens.

\ Keywords: political legitimacy, rationality, methodological individualism, political system, politics 


\section{Introducción}

La legitimidad ha sido para la ciencia sociales un amplio concepto de discusión, reflexión empírica y filosófica, se le asocia a la aceptación y obediencia de los ciudadanos a un determinado esquema institucional, se puede decir que un régimen es legítimo cuando los ciudadanos consideran que las instituciones políticas, los actores y los incentivos que generan, pese a sus falencias son mejores en relación a otros.

En ese sentido, el trabajo toma en consideración la perspectiva teórica de Almond y Verba (1970), que postulan la existencia de orientaciones hacia objetos políticos, para ello señalan tres: 1) evaluativa, 2) afectiva; $y, 3$ ) cognitiva, la primera define valoraciones positivas o negativas de los ciudadanos, la siguiente, refiere el apego a los valores del sistema, mientras que la tercera indica nivel de conocimiento político. No obstante, a esta corriente se le suma las aportaciones de David Easton (1999), sugiere que la legitimidad se puede medir por medio de los apoyos difusos y específicos, el primero apunta la simpatía y lealtad a los principios del régimen, y es independiente del cálculo racional, mientras el segundo indica aceptación o rechazo a las instituciones políticas y sus actores, ambas perspectivas se complementan.

Sin embargo, dada la especificidad de la investigación se tomó en consideración factores socioeconómicos, cómo el ingreso mensual, la situación laboral, la edad, la importancia de la religión, y la escolaridad, etc., con el objetivo de determinar si existe alguna relación estadísticamente significativa con la legitimidad del sistema político, el respaldo empírico para tal relación se encontró en trabajos de reciente publicación como los de, Booth \& Seligson (2009), Fernandes et al (2017) y Somuano (2020), cabe destacar que esos trabajos resaltan el papel determinante que juegan aspectos socioeconómicos en el apoyo a las instituciones de gobierno.

De esta forma, el sistema político guatemalteco se configuro a partir del golpe de Estado del 23 de marzo de 1982; no obstante, resalta su incapacidad en responder las demandas societales (Figura 1 y 2), y paradójicamente, su capacidad de mantenerse y reproducirse en el tiempo, pese a la existencia de un rechazo generalizado a las instituciones políticas. Esas características se resumen en: 1) individuos identificados con los principios del régimen; 2) rechazo a la forma en que el sistema canaliza y procesa las diversas demandas.

Esa contradicción, se estima aquí es de cálculo racional, pues el sistema político guatemalteco no genera suficientes incentivos para alcanzar un nivel óptimo de legitimidad, ni el extremo contrario el cambio de sistema, así, el rechazo y a la vez sostenimiento de un sistema deslegitimado, es producido por la irracionalidad racional, deficiencia cognitiva en la cual se acepta y reconoce de forma racional un curso de acción irracional, por ejemplo, entre ver televisión o formarse políticamente se preferirá lo primero, pues implica una menor inversión de recursos escasos, bajo este esquema el costo de la irracionalidad es menor, y el de la racionalidad mayor. 
Entonces, ser irracional en política es lo más eficiente desde el análisis de coste y rendimiento. Así la escuela de la elección publica o public choice, presenta un esquema analítico sustentado en el individualismo metodológico y la microeconomía, que se puede sintetizar en el supuesto homoeconomicus, individuo racional y maximizador de utilidad, se asume que ese comportamiento (racional y maximizador) se extiende a procesos políticos como el de la legitimidad (Buchanan \& Brennan, 1985; Buchanan \& Tullock, 1999).

De tal forma, la indagación se sustentó con base en las siguientes interrogantes, ¿Cómo se explica desde la racionalidad individual la correlación entre la variable factores socioeconómicos y legitimidad política en Guatemala, período 2019?; es de mencionar, que dada la dinámica del proceso de investigación se dio la posibilidad de plantear una interrogante secundaria, ¿por qué, los ciudadanos guatemaltecos se aferran a sistemas políticos inefectivos?

\section{Materiales y métodos}

La investigación es cuantitativa, no experimental y su alcance correlacional-causal. Se compone por la variable independiente $(\mathrm{x}$ ) denominada factores socioeconómicos, mientras que la variable dependiente (y) legitimidad política. Las unidades de medida fueron de tipo escalarcuantitativas.

En ese sentido, se tomó como base el estudio de cultura política de los guatemaltecos realizado por el Proyecto de Opinión Pública de América Latina (LAPOP), que consistió en una muestra aleatoria estratificada de 1,596 encuestas, hechas a lo largo del país. Un total de 804 individuos fueron encuestados en áreas urbanas y 792 en zonas rurales. El margen de error estimado para la encuesta es de \pm 2.5 .

Las variables se componen por los siguientes indicadores, para la variable independiente, edad (q2); urbano/rural; confianza interpersonal (itl); ingreso mensual del hogar (q10new); percepción de la situación económica personal (idio2); percepción de la situación económica del país (soct2); situación laboral (ocup4a); nivel educativo (ed); e, importancia de la religión (q5b).

Mientras, la variable dependiente por, orgullo por el sistema político (b4); apoya al sistema político (b6); confianza en los partidos políticos (b21); confianza en la Corte Suprema de Justicia (b31); confianza en el Congreso (b13); confianza en la Municipalidad (b32); interés en la política (pol1); y percepción sobre el nivel de conocimiento político (conocim).

Así las variables fueron correlacionadas entre sí, por medio de ocho modelos de regresión lineal, $Y_{t}=B_{0}+B_{1} x+e$; (e = error o residual), además, de técnicas de análisis univariado de datos como, la media y la desviación estándar las cuales permitieron deducir conclusiones de cada uno de los indicadores que componen las variables. 


\section{Resultados}

Las principales características de la cultura política de los guatemaltecos se determinaron a partir del análisis univariado descriptivo y multivariante, a continuación, se visualizan dos figuras en las cuales se presentan los apoyos difusos y específicos de la población estudiada.

\section{Figura 1}

Orgullo y se debería apoyar el sistema político

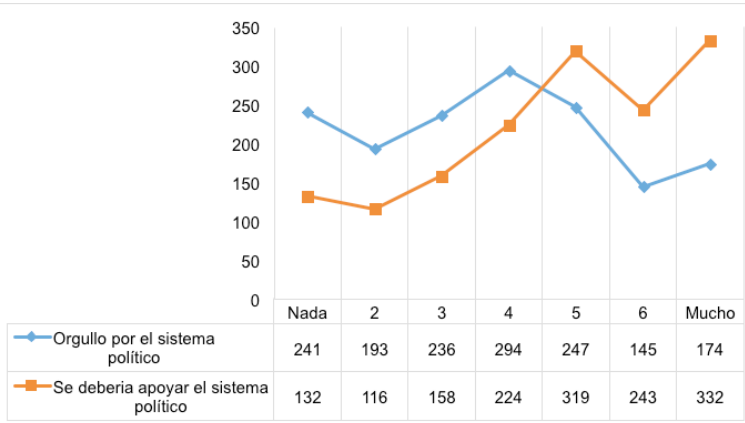

Fuente: Elaboración propia con base en (LAPOP, 2019)

La figura visualiza el apoyo difuso al sistema político guatemalteco, la escala de medida es entre uno y siete, el primero significa nada, mientras que el extremo superior indica mucho. En ese sentido, el indicador representado con la línea color naranja, se debería apoyar el sistema político, arroja los siguientes resultados, la media es de cuatro, mientras la desviación estándar de 1.89.

Eso quiere decir que la mayor cantidad de respuestas se ubican entre el rango de cuatro a siete, la desviación estándar al indicar un valor menor a la media sugiere que las respuestas se encuentran agrupadas y son homogéneas. Puede deducirse que los individuos sienten

apego, pertenencia y lealtad a los principios y valores del sistema político.

En relación al indicador ilustrado con la línea de color azul, orgullo por el sistema político, indica una media de cinco, y una desviación estándar de 1.88, lo cual muestra que las respuestas de los sujetos estudiados igualmente son homogéneas, puede derivarse la existencia de lealtad y devoción por el sistema político.

\section{Figura 2}

Confianza en los partidos políticos, Corte Suprema de Justicia, Municipalidad y Congreso de la República

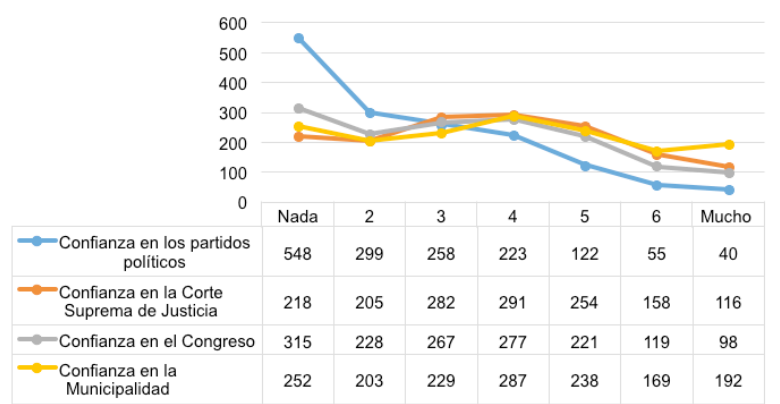

Fuente: Elaboración propia con base en (LAPOP, 2019) 


\section{Tabla 1}

Resumen de los modelos de regresión lineal

\begin{tabular}{|c|c|c|c|c|c|c|c|c|c|c|c|}
\hline & $\mathbf{R}^{2}$ & $q 2$ & itl & q10new & idio2 & ocup4a & soct2 & $q 5 b$ & cp6 & ed & \\
\hline & & & \multicolumn{9}{|c|}{$\begin{array}{l}\text { Variable independiente } \\
\text { (Factores socioeconómicos) }\end{array}$} \\
\hline & & & \multicolumn{9}{|c|}{ Coeficientes de Beta } \\
\hline \multirow{8}{*}{ 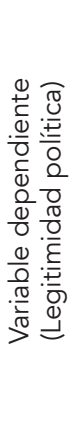 } & b4 & $7 \%$ & -.010 & -.121 & -.036 & $\mathrm{n} / \mathrm{s}$ & $\mathrm{n} / \mathrm{s}$ & -.242 & -.258 & $\mathrm{n} / \mathrm{s}$ & -.068 \\
\hline & b6 & $3 \%$ & $\mathrm{n} / \mathrm{s}$ & $\mathrm{n} / \mathrm{s}$ & $\mathrm{n} / \mathrm{s}$ & $\mathrm{n} / \mathrm{s}$ & $\mathrm{n} / \mathrm{s}$ & $\mathrm{n} / \mathrm{s}$ & -.416 & $\mathrm{n} / \mathrm{s}$ & $\mathrm{n} / \mathrm{s}$ \\
\hline & b21 & $5 \%$ & -.010 & -.093 & $n / s$ & $n / s$ & $\mathrm{n} / \mathrm{s}$ & -.235 & -.277 & $\mathrm{n} / \mathrm{s}$ & -.048 \\
\hline & b31 & $7 \%$ & $n / s$ & -.212 & $n / s$ & $n / s$ & $\mathrm{n} / \mathrm{s}$ & -.282 & -.405 & $\mathrm{n} / \mathrm{s}$ & $\mathrm{n} / \mathrm{s}$ \\
\hline & b13 & $11 \%$ & -.0133 & -.148 & -.027 & $\mathrm{n} / \mathrm{s}$ & $\mathrm{n} / \mathrm{s}$ & -.351 & -.393 & $\mathrm{n} / \mathrm{s}$ & -.086 \\
\hline & b32 & $5 \%$ & $n / s$ & -.183 & $\mathrm{n} / \mathrm{s}$ & -.232 & $\mathrm{n} / \mathrm{s}$ & $n / s$ & -.260 & $\mathrm{n} / \mathrm{s}$ & -.065 \\
\hline & pol1 & $5 \%$ & .007 & .072 & -.019 & $\mathrm{n} / \mathrm{s}$ & $\mathrm{n} / \mathrm{s}$ & $\mathrm{n} / \mathrm{s}$ & $\mathrm{n} / \mathrm{s}$ & $\mathrm{n} / \mathrm{s}$ & $\mathrm{n} / \mathrm{s}$ \\
\hline & conocim & $23 \%$ & -.003 & $\mathrm{n} / \mathrm{s}$ & -.022 & $\mathrm{n} / \mathrm{s}$ & .029 & $\mathrm{n} / \mathrm{s}$ & $\mathrm{n} / \mathrm{s}$ & $\mathrm{n} / \mathrm{s}$ & -.074 \\
\hline
\end{tabular}

Fuente: Elaboración propia con base en (LAPOP, 2019)

Por otra parte, la tabla 1 agrupa ocho modelos de regresión los cuales son significativos con un valor de $\mathrm{P}<0.05$; los indicadores que no presentan correlación se les agrego la sigla de n/s que indica no existe relación estadísticamente significativa. La forma correcta de leer la tabla es de izquierda a derecha, en la primera columna aparecen las abreviaturas de la variable dependiente, en la siguiente se visualiza el coeficiente de determinación $\left(R^{2}\right)$, mientras que en las columnas resaltadas con color negro se señalan las siglas de los indicadores de la variable independiente, los números que se ubican en las columnas resaltadas con color gris representan los coeficientes de beta, muestran la dirección en que se relacionan las variables.

Así, cuando la variable dependiente es orgullo por el sistema político, los resultados indican que los más jóvenes sienten un mayor orgullo por el sistema, los individuos con una alta confianza interpersonal igualmente se enorgullecen, al igual que los sujetos pertenecientes a familias con un ingreso mensual reducido y los altamente religiosos.

Asimismo, sí la variable dependiente es, se debería apoyar el sistema político, los predictores que arrojan relación estadísticamente significativa son, la edad y la importancia de la religión, eso indica que los jóvenes y las personas que consideran la religión como parte fundamental de sus vidas consienten el sistema. En consecuencia, cuando la variable dependiente es interés en la política los individuos de mayor edad, con menor confianza interpersonal, de menor ingreso mensual familiar fueron quienes no presentan interés en dicha actividad. 
Igualmente, al representar la variable dependiente con nivel de conocimiento político, se determinó que, los individuos de menor edad, menor ingreso, los desempleados, y los que tienen menos años de escolaridad saben menos sobre política.

\section{Discusión}

En cuanto a los modelos de regresión se puede deducir que las personas jóvenes, con arraigo religioso, menos ingreso mensual familiar, con una visualización negativa de la situación económica del país y de menor escolaridad sienten arraigo por el sistema político y sus instituciones.

Podría suponerse que el problema de la legitimidad del sistema político guatemalteco, se resuelve mejorando los ingresos, la situación económica del país o aumentando la escolaridad, bajo este esquema se asumiría que los individuos no apoyarían un entramado institucional como el guatemalteco. Sin embargo, se estima que esa argumentación es trivial, pues la problemática está vinculada a los fallos sistémicos de la política, es decir la imposibilidad de repartir bienes públicos de forma eficiente y a la racionalidad individualidad ligada al homoeconomicus, en tanto el cálculo racional del mercado se reproduce en los procesos político, de forma simétrica tal y como lo postulan los economistas James Buchanan y Gordon Tullock (1999).

En ese sentido, la política democrática implica la violación del óptimo de Pareto, esto ocurre porque los intereses y objetivos de los individuos son dispares al igual que sus necesidades, por tal motivo, no es concebible que una sola mente humana diseñe una política pública que satisfaga todas las preferencias individuales, de esta forma se genera un cíclico descontento hacia las instituciones políticas.

Así se presentan cuatro paradojas, que explican porque los individuos legitiman sistemas políticos deficientes, siendo estas, paradoja de la información y la irracionalidad racional, paradoja fatalista, paradoja benevolente, y; paradoja de la intolerancia.

La primera consiste en una deficiencia cognitiva, entendida como la acción de ser conscientemente irracional en el ámbito político dado el alto costo de la información y contrariamente, el bajo costo de la irracionalidad, en resumen, en política el error no genera cuantiosas pérdidas de recursos escasos, es más racional desde el punto de vista de la maximización de utilidad ser un irracional político, así fácilmente los individuos pueden aceptar y promover ideas irracionales y absurdas.

En seguida, se presentó la paradoja fatalista, que no es más que una tendencia permanente a creer que las instituciones políticas siempre funcionan mal, esto ocurre porque el gobierno no puede satisfacer una función de utilidad que represente los deseos globales de la comunidad, eso hace que una buena parte de los individuos se mantengan inconformes con las decisiones y políticas públicas. 
A diferencia del mercado, ese sí raparte bienes con eficiencia, pues al realizar un intercambio de forma voluntaria entre dos individuos ocurre un acuerdo unánime (ambos acordaron vender y comprar un recurso escaso), esa unanimidad no es posible alcanzarla en la repartición de bienes públicos, pues ese escenario forzosamente afecta la generación de riqueza y derechos de propiedad de determinados individuos.

Así, la paradoja benevolente, indica que los individuos tienen un sentimiento moral a procurar el bienestar común o al menos de forma cognitiva, en ese sentido, es natural instar a los políticos a aumentar las funciones y actividades del gobierno, sin embargo, ese sentir moral no se guía por el cálculo racional, pues, en política los individuos promueven las ideas que les hacen sentir reconfortados, de esta manera dicha incoherencia se puede definir como la propensión natural de los ciudadanos a procurar una mayor intervención gubernamental en la reducción de la pobreza y equiparación del ingreso.

Se estima que estas tres incongruencias se presentan de forma más o menos coherente en la mayoría de sistemas democráticos occidentales, de tal forma, existe una paradoja propia de la sociedad guatemalteca, se refiere a la intolerancia, radica en una adopción de principios democráticos únicamente por la popularidad de aquella forma de gobierno y a su vez conlleva rechazar prácticas como la diversidad sexual, cultural o religiosa.
Finalmente, los sistemas políticos inmersos en las reglas de la democracia liberal acarrean dificultades para satisfacer las preferencias y deseos de los ciudadanos, esto sucede porque la repartición de bienes públicos no se da bajo un acuerdo unánime, además persiste la imposibilidad de establecer una función de utilidad que represente a toda la colectividad, causándose así una permanente crisis de legitimidad. Pues siempre existirán minorías - grupos de presión que impondrán sus proyectos al resto de la población, por medio de las populares reglas democráticas.

\section{Agradecimientos}

Se agradece al proyecto de Opinión Pública de América Latina (LAPOP) y a sus principales donantes (la Agencia de los Estados Unidos para el Desarrollo Internacional, el Programa de las Naciones Unidas para el Desarrollo, el Banco Interamericano de Desarrollo y Vanderbilt University) por poner a disposición los datos. Así como a todos los lectores del artículo. 


\section{Referencias}

Almond, G., \& Verba, S. (1970). La cultura civica. Estudio sobre la participación política democrática en cinco naciones. (José Belloch Ed y Trad). Euramerica, S.A.

Booth, J. A., \& Seligson, M. A. (2009). The Legitimacy Puzzle in Latin America: Political Support and Democracy in Eight Nations. Cambridge University Press. https://doi.org/10.1017/CBO9780511818431

Buchanan, J., \& Brennan, G. (1985). La razon de las normas. Economía política constitucional. Unión Editorial.

Buchanan, J., \& Tullock, G. (1999). The Calculus of Consent: Logical Foundations of Constitutional Democracy. Liberty Fund.

Easton, D. (1999). Esquema para el análisis político (Ricardo Murtagh Ed). Amorrortu.

Fernandes, L., Nicolás, M., Ribeiro, E., Nicolás, M., \& Callai, R. (2017). El efecto de la experiencia democrática en la estructura de la legitimidad en América Latina y el Caribe. Opinao Publica, 23(2), 289-315. http://dx.doi.org/10.1590/1807 01912017232289

LAPOP. (2019). Base de datos: «Guatemala». Programa de opinión pública de América Latina, Universidad de Vanderbilt. http://datasets. americasbarometer.org/database/index.php?freeUser=true

Somuano, Ma. F. (2020). Los determinantes dela legitimidad gubernamental: El sexenio de Enrique Peña Nieto. Foro Internacional, 60(2), 367396. https://doi.org/10.24201/fi.v60i2.2729 


\section{Sobre el autor}

Es politicólogo con estudios de maestría en investigación social, su trabajo académico ha girado en torno al estudio de la racionalidad individual, individualismo metodológico, y el análisis de cómo los gobiernos procesan las divergentes preferencias individuales; además, ha sido investigador y profesor para la Universidad de San Carlos de Guatemala y consultor en temas legislativos para organizaciones internacionales.

Copyright (c) José Ernesto Javier Duarte Madrid

Este texto está protegido por una licencia Creative Commons 4.0.

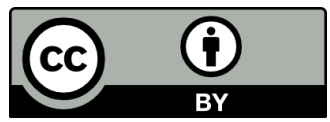

Usted es libre para compartir, copiar y redistribuir el material en cualquier medio o formato y adaptar el documento, remezclar, transformar y crear a partir del material para cualquier propósito, incluso comercialmente, siempre que cumpla la condición de atribución: usted debe reconocer el crédito de una obra de manera adecuada, proporcionar un enlace a la licencia, e indicar si se han realizado cambios. Puede hacerlo en cualquier forma razonable, pero no de forma tal que sugiera que tiene el apoyo del licenciante o lo recibe por el uso que hace. 